\title{
Politique
}

Politique

\section{Donat J. TADDEO et Raymond C. TARAS, Le débat linguistique au Québec, Montréal, Presses de l'Université de Montréal, 1987, (traduit de l'anglais par Brigitte Morel-Nish)}

\section{Hubert Guindon}

Numéro 17, hiver 1990

Les nouveaux enjeux du politique

URI : https://id.erudit.org/iderudit/040658ar

DOI : https://doi.org/10.7202/040658ar

Aller au sommaire du numéro

Éditeur(s)

Société québécoise de science politique

ISSN

0711-608X (imprimé)

1918-6584 (numérique)

Découvrir la revue

Citer ce compte rendu

Guindon, H. (1990). Compte rendu de [Donat J. TADDEO et Raymond C. TARAS, Le débat linguistique au Québec, Montréal, Presses de l'Université de Montréal, 1987, (traduit de l'anglais par Brigitte Morel-Nish)]. Politique, (17), 176-179.

https://doi.org/10.7202/040658ar d'utilisation que vous pouvez consulter en ligne.

https://apropos.erudit.org/fr/usagers/politique-dutilisation/ 
Donat J. TADDEO et Raymond C. TARAS, Le débat linguistique au Québec, Montréal, Presses de l'Université de Montréal, 1987, (traduit de l'anglais par Brigitte Morel-Nish).

Le sujet dont traite ce volume est indiqué non pas dans le titre mais dans le sous-titre qui n'apparaît pas sur la page couverture: «La communauté italienne et la langue d'enseignement». En plus de décrire, de manière chronologique, les efforts répétés de la communauté italienne de Montréal pour obtenir et maintenir des écoles catholiques bilingues, ce livre identifie les associations et les chefs de file qui se sont succédé comme porte-parole de la communauté italienne pour réclamer de telles écoles. Plus intéressant encore est le dévoilement des stratégies et des tactiques que le Consiglio Educativo Italo-Canadese (composé de «jeunes turcs» italiens) a utilisées pour déloger ceux qui les ont précédés comme porte-parole de la communauté italienne: la CIPBA (Canadian Italian Professional and Businessmen's Association) et ensuite la FAIQ (Fédération des associations italiennes du Québec) fondé par Pietro Rizzuto, maintenant sénateur libéral. On aura noté que l'on passe de l'anglais, au français, à l'italien.

Le Consiglio se détacha de la FAIQ de Rizzuto parce qu'il avait été manipulé et aussi parce que «le groupe avait surtout le sentiment qu'il n'était plus possible de se fier aux organisations communautaires dirigées par des représentants des hommes d'affaires, plus orientés vers leurs propres intérêts que vers celui de la communauté» (p. 149). La plupart des seize membres fondateurs du Consiglio «avaient été éduqués au Québec dans le système anglais, dont certains faisaient partie aujourd'hui comme directeurs ou enseignants». Leur opposition au Bill 22 se basait sur les conséquences que ce dernier aurait sur la communauté italienne. «Bien sûr», nous avoue l'auteur principal, «ce souci a aussi pu être dicté par l'intérêt de ceux qui enseignaient dans les écoles anglaises élémentaires de l'île de Montréal» (ibid.).

L'intérêt de la communauté italienne était «évidemment» de contourner la loi 22 et le Consiglio s'évertua, dans les sous-sols des églises italiennes, à préparer les enfants d'âge pré-scolaire aux fameux tests linguistiques qui leur permettraient de s'inscrire à 
l'école anglaise. En février 1975, 96 professeurs travaillaient dans des classes «clandestines» auprès de 869 élèves dans les sous-sols de six églises (p. 151). Le Consiglio «organisa des classes clandestines pour près de 1500 enfants» (p. 153).

Cette mobilisation du Consiglio eut un double effet: celui d'en faire le porte-parole de la communauté italienne au lieu du FAIQ de Rizzuto et de compliquer la vie au gouvernement libéral au pouvoir.

Lors des élections de 76, le Consiglio «s'engagea donc résolument dans l'arène politique. Il déclara sans ambages qu'à l'avenir le Parti libéral ne devrait plus compter sur l'appui inconditionnel de la communauté italienne...» (p. 187). À un débat politique organisé par le Consiglio avec les trois partis politiques devant 1500 personnes, le représentant du Parti libéral, Fernand Lalonde fut hué. Plus tard, à la suite de l'adoption de la loi 101, des enseignants aidèrent les parents à faire de fausses déclarations pour que leurs enfants puissent continuer à fréquenter les écoles anglaises.

Les frais de voyage du «Moi glorieux» (Ego-trips) sont toujours considérables. Dans ce cas-ci, ironiquement, le Consiglio contribua à faire élire le Parti québécois, ce qui n'était évidemment pas son objectif. Il disparut de la carte pour être remplacé par le Congresso. Certains de leurs membres ont refait surface aux élections de 1985, comme députés libéraux et ont obtenu, par l'entremise de Claude Ryan, devenu ministre de l'Éducation, l'amnistie générale des illégaux, c'est-à-dire l'acceptation officielle à l'école anglaise (p. 209). Ce qu'entendent les auteurs par intégration est pleinement rendu par la dernière phrase du volume: La présence de Macchiagodena et de Cusano au sein du comité Rondeau de même que celle de Cusano, de Macciocia et de Ciaccia, ministre de l'Énergie, à la députation provinciale libérale, prouvait bien qu'en 1986 la communauté italienne était solidement intégrée à la société québécoise et qu'elle n'avait plus autant besoin de groupes de pression pour se faire valoir. À cette liste, il faudrait évidemment ajouter le nom de Taddeo devenu, depuis, le délégué du Québec à Rome. 
C'est avec un malin plaisir que l'auteur principal, ex-doyen à l'Université Concordia et ancien commissaire à la CECM, nous raconte en détail les péripéties du Consiglio avec le Parti libéral d'abord, avec la CECM ensuite, moins complaisante après 1976. La candeur des propos sur l'attitude de la communauté italienne face à la langue d'enseignement constitue un dossier politique intéressant et éclairant. Cette monographie sur l'idéologie, les stratégies et les tactiques de la communauté italienne est intéressante à plusieurs égards.

En revanche, il s'agit d'une contribution décevante au «débat linguistique au Québec». Les auteurs proposent aux Québécois d'imiter les Italiens en devenant bilingues: La CECM eut aussi l'occasion unique de transformer radicalement tout le système d'éducation catholique de la ville et n'en prit pas plus avantage. En juillet 1940 un commissaire anglophone présenta une motion par laquelle il demandait l'adoption d'un programme entièrement bilingue pour toutes les écoles dans la juridiction de la Commission (p. 54). Voilà l'origine de nos problèmes, de conclure les auteurs: Si, en 1940, la CECM avait saisi l'occasion qui se présentait de relever le statut de la langue française, surtout en l'enseignant aux enfants allophones, il n'aurait peut-être pas été nécessaire d'en venir aux mesures contraignantes des années 70 (p. 55). Aux États-Unis, cela s'appelle du transitional bilingualism, une concession faite parfois aux immigrants en voie d'assimilation. Le prix à payer par l'immigrant qui veut s'intégrer est non seulement l'obligation d'apprendre la langue du pays qui l'accueille mais également celui de perdre petit à petit la langue de ses ancêtres. Proposer que le Québécois se comporte en immigrant au Québec, voilà où en arrivent Taddeo et Taras, tous deux Montréalais d'origine, produits des écoles et collèges anglo-catholiques.

Heureusement toutefois, une nouvelle étude sur les allophones est maintenant disponible. Elle est faite par une Montréalaise d'origine italienne, Ines Molinaro. Également diplômée de l'Université Concordia, sa thèse de doctorat (qui s'intitule: Conquest and Reconquest: the Quebec Political Culture) constitue une analyse brillante des conséquences de la Révolution tranquille sur les allophones en général et sur les Italiens en particulier. Son tra- 
vail aborde une dimension ignorée de Taddeo et Taras et constitue, pour le lecteur sérieux, un complément indispensable.

Hubert Guindon Université Concordia 